\title{
Self-Interference Distribution over Full-Duplex Multi-User MIMO Channels
}

\author{
Arman Shojaeifard*, Kai-Kit Wong*, Marco Di Renzo ${ }^{\dagger}$, Khairi Ashour Hamdi ${ }^{\ddagger}$, and Jie Tang ${ }^{\S}$ \\ *Communications and Information Systems Group, University College London, London, United Kingdom \\ †Laboratoire des Signaux et Systémes, CNRS, CentraleSupélec, Univ Paris Sud, Université Paris-Saclay, Paris, France \\ $\ddagger$ Microwave and Communication Systems Group, University of Manchester, Manchester, United Kingdom \\ $\S$ Smart Information Processing Centre, South China University of Technology, Guangzhou, China \\ E-Mail: *a.shojaeifard@ucl.ac.uk; *kai-kit.wong@ucl.ac.uk; †marco.direnzo@12s.centralesupelec.fr; \\ $\ddagger$ k.hamdi@manchester.ac.uk; §eejtang@scut.edu.cn
}

\begin{abstract}
We consider the case where a reference full-duplex (FD) node (e.g., base station), equipped with arbitrary number of transmit/receive antennas, utilizes generalized linear beamformers to simultaneously communicate with multiple FD radios (e.g., user equipments). The fading coefficients for the residual self-interference (SI) channels are drawn from the complex Gaussian distribution with arbitrary mean and variance. Here, it is not possible to directly derive the distribution of the bidirectional channel power gain. As a result, we adopt the method of moments in order to provide a new Gamma approximation for the residual SI distribution over FD multi-user MIMO Rician fading channels. The proposed theorem holds under arbitrary linear precoder/decoder design, number of antennas and streams, and SI cancellation capability. The validity of the theoretical findings is confirmed via extensive simulations of the entire transmit/receive processing chain.
\end{abstract}

\section{INTRODUCTION}

The emergence of multiple-input multiple-output (MIMO) technology has revolutionized wireless communications [1], [2]. MIMO provides entirely new degrees of freedom, allowing for the implementation of key technologies and techniques such as diversity and multiplexing in the spatial domain. As a result, significant performance improvements have been achieved through exploiting MIMO in modern wireless systems. This trend is expected to continue going forward, with the emergence of new MIMO architectures such as the largescale antenna system (LSAS) [3], [4].

Existing wireless systems have been designed and dimensioned under a strict separation of the transmit and the receive functions, referred to as half-duplex (HD) mode [5]. This is typically achieved through orthogonal radio frequency (RF) partitioning, such as time-division duplex (TDD) and frequency-division duplex (FDD), where the different communications directions occur over distinct time slots and frequency bands, respectively. The motivation behind HD design has largely been to avoid the overwhelming self-interference (SI), which arises from the bi-directional operation [6].

With the rapid increase in traffic, under limited RF resources, however, there has been an enormous incentive for an overhaul in the wireless system architecture [7]. A prominent candidate solution involves the transmission and reception of information over the same RF resources, i.e., full-duplex (FD) mode [8], [9]. There is a growing surge of interest in this topic, as in theory, FD has the potential to double the spectral efficiency compared to HD. This trend is in line with recent major advances in SI supression techniques [10]-[12]. Broadly speaking, SI can be tackled using isolation in space [13], [14], digital/analog subtraction [15], [16], and as a combination of both [17]. Several real-time point-to-point prototypes of FD radio nodes have been demonstrated in practice recently [18].

Despite pioneering efforts, the study of FD mode in multiuser MIMO setups remains an open problem [19]. Most existing works in the literature rely on simplifying assumptions, particularly concerning the SI. A long-standing limitation involves using the Rayleigh distribution for the bi-directional fading channel model [20]-[22]. This approach, whilst improving on the common perfect SI cancellation assumption, differs from experimental measurements which have shown that the residual SI channel undergoes Rician fading [10], [18]. As a result, a rigorous characterization of the residual SI statistics over FD multi-user MIMO Rician fading channels is critical for facilitating performance analysis and optimization.

In this work, we focus on a reference FD multi-antenna node functioning as a transmitter and a receiver with respect to an arbitrary number of FD terminals over the same time and frequency. The complex Gaussian distribution is accordingly employed in order to capture the characteristics of the residual SI under arbitrary SI cancellation capability. By applying linear precoding and decoding, it is not feasible to derive the statistics of the bi-directional channel power gain directly. As a result, we exploit the method of moments in order to develop a unified mathematical expression for the distribution of the residual SI over FD multi-user MIMO Rician fading channels. The theoretical findings are validated via extensive simulations of the entire RF transmit/receive processing chain.

Notation: $\boldsymbol{X}$ is a matrix; $\boldsymbol{x}$ is a vector; $T, \dagger$, and + are the transpose, conjugate-transpose, and pseudo-inverse operations; $\mathbb{E}\{$.$\} is the expected value; \operatorname{Var}\{$.$\} is the variance; \mathcal{P}($.$) is the$ probability density function (pdf); |.| is the modulus; $\|$.$\| is the$ norm; $I_{0}($.$) is the zeroth-order Bessel function of the first kind,$ respectively. 


\section{SySTEM MODEL}

We consider the general scenario where a FD multi-antenna node is transmitting and receiving information with respect to multiple FD radios over the same time and frequency. We use $M$ and $N$ in order to denote the arbitrary number of RF transmit and receive chains at the reference FD multi-antenna node, respectively. The arbitrary number of FD radios, i.e., transmit/receive streams, is in turn represented with $U$. An immediate application example that can be highlighted here is a reference base station which communicates simultaneously in the downlink and the uplink with multiple user equipments, in FD mode. For simplicity, we assume $U<\min (N, M)$ holds, hence avoiding the need for channel assignment.

Let $\boldsymbol{g}_{k} \in \mathcal{C}^{1 \times M}$ and $\boldsymbol{h}_{k} \in \mathcal{C}^{N \times 1}$ denote the transmit and receive fading channels of the respective FD multi-antenna node with respect to the $k$-th FD radio, $k \in \mathcal{U}=\{1, \cdots, U\}$. Further, the corresponding combined channels are respectively $\boldsymbol{G}=\left[\boldsymbol{g}_{k}^{T}\right]_{1<k<U}^{T} \in \mathcal{C}^{U \times M}$ and $\boldsymbol{H}=\left[\boldsymbol{h}_{k}\right]_{1 \leq k \leq U} \in \mathcal{C}^{N \times U}$. Moreover, the cross-mode fading channel at the $k$-th FD radio from the $j$-th FD radio is denoted with $q_{k j}, j \in \mathcal{U} \backslash\{k\}$. Further, $\boldsymbol{F}_{0} \in$ $\mathcal{C}^{N \times M}$ and $f_{k}$ denote the residual SI channels at the reference FD multi-antenna node and the $k$-th FD radio, respectively. The residual SI channels, $\boldsymbol{F}_{0}$ and $f_{k}$, are subject to Rician fading with independent and identically-distributed elements drawn from a complex Gaussian distribution $\mathcal{C N}\left(\mu, \nu^{2}\right)$ [23]. All other communications channels are assumed to undergo Rayleigh fading with independent and identically-distributed elements drawn from $\mathcal{C N}(0,1)$ [24]. We assume channel state information (CSI) is available throughout.

In this work, we consider arbitrary linear beamforming design at the transmitter and the receiver of the reference FD multi-antenna node. Let $s=\left[s_{k}\right]_{1 \leq k \leq U}^{T} \in \mathcal{C}^{U \times 1}$ denote the corresponding complex information vector of the reference node with respect to the neighboring sources (in either direction). Hence, the transmit signal vector under linear precoding at the reference FD multi-antenna node is constructed as $\boldsymbol{t}=\boldsymbol{V} \boldsymbol{s}$ where $\boldsymbol{V}=\left[\boldsymbol{v}_{k}\right]_{1 \leq k \leq U} \in \mathcal{C}^{M \times U}$ is the precoding matrix. Further, the linear receive filter at the reference FD multiantenna node is denoted using $\boldsymbol{W}=\left[\boldsymbol{w}_{k}^{T}\right]_{1 \leq k \leq U}^{T} \in \mathcal{C}^{U \times N}$.

The received signal from the reference FD multi-antenna node at an arbitrary $\mathrm{FD}$ radio $k^{*}, k^{*} \in \mathcal{U}$, is given by

$$
\begin{gathered}
x_{k^{*}}=\underbrace{\sqrt{p_{0}} \boldsymbol{g}_{k^{*}} \boldsymbol{v}_{k^{*} s_{k^{*}}}}_{\text {useful signal, } d-k^{*}}+\underbrace{\sqrt{p_{0}} \sum_{k=1, k \neq k^{*}}^{U} \boldsymbol{g}_{k} \boldsymbol{v}_{k} s_{k}}_{\text {multi-user interference, } d d-k^{*}} \\
+\underbrace{\sum_{k=1, k \neq k^{*}}^{U} \sqrt{p_{k}} q_{k^{*} k^{*}} s_{k}}_{\text {cross-mode interference, } u d-k^{*}}+\underbrace{\sqrt{p_{k^{*}} f_{k^{*}} s_{k^{*}}}}_{\text {self-interference, } d s i-k^{*}}+\underbrace{\eta_{k^{*}}}_{\text {noise }}
\end{gathered}
$$

where $p_{0}$ is the reference FD multi-antenna node per-stream transmit power, $p_{k}$ is the $k$-th FD radio transmit power, and $\eta_{k^{*}}$ is the complex additive white Gaussian noise (AWGN), with mean zero and variance $\sigma_{d}^{2}$, at the $k^{*}$-th FD radio, respectively. Next, the post-processing received signal from the $k^{*}$ FD radio at the reference FD multi-antenna node is given by

$$
\begin{gathered}
y_{k^{*}}=\underbrace{\sqrt{p_{k^{*}}} \boldsymbol{w}_{k^{*}}^{T} \boldsymbol{h}_{k^{*} s_{k^{*}}}}_{\text {useful signal, } u-k^{*}}+\underbrace{\sum_{\text {self-interference, } u s i-k^{*}}^{U} \sqrt{p_{k}} \boldsymbol{w}_{k}^{T} \boldsymbol{h}_{k} s_{k}}_{\text {multi-user interference, } u u-k^{*}} \\
+\underbrace{\sqrt{p_{0}} \boldsymbol{w}_{k^{*}}^{T} \boldsymbol{F}_{0} \boldsymbol{V} \boldsymbol{s}}_{\text {scaled noise }}+\underbrace{\boldsymbol{w}_{1, k}^{T}}_{k^{*} \boldsymbol{\eta}}
\end{gathered}
$$

where $\boldsymbol{\eta} \in \mathcal{C}^{N \times 1}$ is the circularly-symmetric complex AWGN vector with mean zero and covariance matrix $\sigma_{u}^{2} \mathbf{I}_{N}$.

\section{Signals Statistics}

The received signal-to-interference-plus-noise ratio (SINR) at the typical FD radio $k^{*}$ from the reference FD multi-antenna node under consideration can be expressed as

$$
\operatorname{SINR}_{x_{k^{*}}}=\frac{\mathcal{X}_{k^{*}}}{\mathcal{I}_{d d-k^{*}}+\mathcal{I}_{u d-k^{*}}+\mathcal{I}_{d s i-k^{*}}+\sigma_{d}^{2}}
$$

where $\mathcal{X}_{k^{*}}=p G_{k^{*}}, \mathcal{I}_{d d-k^{*}}=p_{0} \sum_{k=1, k \neq k^{*}}^{U} G_{k}, \mathcal{I}_{u d-k^{*}}=$ $\sum_{k=1, k \neq k^{*}}^{U} p_{k} Q_{k^{*}-k}$, and $\mathcal{I}_{d s i-k^{*}}=p F_{k^{*}}$ are the different signals involved, with channel power gains $G_{k^{*}} \triangleq\left|\boldsymbol{g}_{k^{*}} \boldsymbol{v}_{k^{*}}\right|^{2}$, $G_{k} \triangleq\left|\boldsymbol{g}_{k} \boldsymbol{v}_{k}\right|^{2}, Q_{k^{*}-k} \triangleq\left|q_{k^{*} k}\right|^{2}$, and $F_{k^{*}} \triangleq\left|f_{k^{*}}\right|^{2}$, respectively. Moreover, the received SINR from the $k^{*}$ FD radio at the reference FD multi-antenna node is formulated as

$$
\operatorname{SINR}_{y_{k^{*}}}=\frac{\mathcal{Y}_{k^{*}}}{\mathcal{I}_{u u-k^{*}}+\mathcal{I}_{u s i-k^{*}}+\sigma_{u}^{2}}
$$

where we have $\mathcal{Y}_{k^{*}}=p_{k^{*}} H_{k^{*}}, \mathcal{I}_{u u-k^{*}}=\sum_{k=1, k \neq k^{*}}^{U} p_{k} H_{k}$, $\mathcal{I}_{\text {usi-k }}{ }^{*}=p F_{0-k^{*}}, H_{k^{*}} \triangleq\left|\boldsymbol{w}_{k^{*}}^{T} \boldsymbol{h}_{k^{*}}\right|^{2}, H_{k} \triangleq\left|\boldsymbol{w}_{k}^{T} \boldsymbol{h}_{k}\right|^{2}$, and $F_{0-k^{*}} \triangleq\left\|\boldsymbol{w}_{k^{*}}^{T} \boldsymbol{F}_{0} \boldsymbol{V}\right\|^{2}$.

With linear processing over MIMO Rayleigh fading channels, the signals statistics can be captured using the Gamma distribution [25]. For example, consider the reference FD multi-antenna node uses linear zero-forcing ( $\mathrm{ZF}$ ) beamforming for removing the multi-user interference in both communications directions by setting (i) the column vectors of the linear precoder $\boldsymbol{V}$ equal to the normalized columns of $G^{+}=$ $\boldsymbol{G}^{\dagger}\left(\boldsymbol{G} \boldsymbol{G}^{\dagger}\right)^{-1}$, and (ii) the row vectors of the linear decoder $\boldsymbol{W}$ equal to the normalized rows of $\boldsymbol{H}^{+}=\left(\boldsymbol{H}^{\dagger} \boldsymbol{H}\right)^{-1} \boldsymbol{H}^{\dagger}$. With the projection of each intended channel vector onto the nullspace spanned by the multi-user interference, the useful channel power gains can be characterized as $G_{k^{*}} \sim \operatorname{Gamma}(M-U+1,1)$ and $H_{k^{*}} \sim \operatorname{Gamma}(N-U+1,1)$. Moreover, the cross-mode interfering channel power gain from the $k$-th FD radio is distributed as $Q_{k^{*}-k} \sim \operatorname{Gamma}(1,1) \equiv \operatorname{Exp}(1), k \in \mathcal{U} \backslash\left\{k^{*}\right\}$.

\section{Self-Interference Distribution}

The exact residual SI distribution at the neighboring FD radios can be readily derived as in the following result.

Lemma 1. Consider the residual SI fading channel, $f_{k}$, of an arbitrary $F D$ radio $k$, with single $R F$ transmit/receive chain, following a complex Gaussian distribution with mean $\mu$ and standard deviation $\nu$. The magnitude of this channel, $\left|f_{k}\right|$, is a Rician random variable with Rician factor $K$ and fading attenuation $\Omega$ where $\mu \triangleq \sqrt{\frac{K \Omega}{K+1}}$ and $\nu \triangleq \sqrt{\frac{\Omega}{K+1}}$. The 
corresponding residual SI channel power gain, $F_{k}=\left|f_{k}\right|^{2}$, is a non-central Chi-squared random variable with $p d f$

$$
\mathcal{P}_{F_{k}}(x)=\frac{1+K}{\Omega} \exp \left(-\left(K+\frac{(1+K) x}{\Omega}\right)\right) I_{0}\left(2 \sqrt{\frac{K(1+K) x}{\Omega}}\right) .
$$

The moment matching technique can be applied here to provide a Gamma approximation for the non-central Chisquared distribution of the residual SI $F_{k}$ in (5).

Lemma 2. The residual SI channel power gain, of an arbitrary $F D$ radio $k$, with single $R F$ transmit/receive chain and fading coefficients drawn from the complex Gaussian distribution with mean $\mu$ and standard deviation $\nu$, can be approximated using $F_{k} \sim \operatorname{Gamma}(\kappa, \theta)$ where

$$
\kappa \triangleq \frac{\left(\mu^{2}+\nu^{2}\right)^{2}}{\left(2 \mu^{2}+\nu^{2}\right) \nu^{2}}
$$

and

$$
\theta \triangleq \frac{\left(2 \mu^{2}+\nu^{2}\right) \nu^{2}}{\mu^{2}+\nu^{2}} .
$$

On the other hand, the residual SI channel power gain at the reference FD multi-antenna node equipped with an arbitrary number of RF transmit/receive chains, involves a sum of nonidentically distributed positive random variables. For example, for the decoding of the $k$-th stream, $F_{0-k}=\left\|\boldsymbol{w}_{k}^{T} \boldsymbol{F}_{0} \boldsymbol{V}\right\|^{2}$ where $\boldsymbol{w}_{k}^{T} \boldsymbol{F}_{0} \boldsymbol{V}=\left[\sum_{i=1}^{N} \sum_{j=1}^{M}\left\{\boldsymbol{w}_{k}^{T}\right\}_{i}\left\{\boldsymbol{F}_{0}\right\}_{i, j}\{\boldsymbol{V}\}_{j, k}\right]_{1<k<U}$.

It is therefore not possible to directly derive the exact distribution of $F_{0, k}, k \in \mathcal{U}$. In this work, we derive closedform expressions for the exact first and second moments of $F_{0-k}, k \in \mathcal{U}$. We then apply the Gamma moment matching technique to develop a unified approximation for the residual SI distribution.

Theorem. Consider the residual SI fading channel, $F_{0}$, of a reference FD multi-antenna node, with $M$ transmit and $N$ receive antennas, following a per-entry complex Gaussian distribution with mean $\mu$ and standard deviation $\nu$. The reference FD multiantenna node applies a linear precoder $\boldsymbol{V}$ and a linear decoder $\boldsymbol{W}$ to simultaneously transmit/receive information streams with respect to $U$ neighboring FD radios. The residual SI channel power gain with arbitrary linear beamforming over FD multi-user MIMO Rician fading channels can be approximated using $F_{0, k} \sim \operatorname{Gamma}(\kappa, \theta), k \in \mathcal{U}=\{1, \cdots, U\}$, where

$$
\kappa \triangleq \frac{U(N+1)(M-U+2)\left(\mu^{2}+\nu^{2}\right)^{2}}{\left(2 N M+\frac{U(M-U+2)}{(M+1)}(N M-N-M-1)\right) \mu^{4}+(N+1)(M+1) \nu^{2}\left(2 \mu^{2}+\nu^{2}\right)}
$$

and

$$
\theta \triangleq \frac{\left(2 N M+\frac{U(M-U+2)}{(M+1)}(N M-N-M-1)\right) \mu^{4}+(N+1)(M+1) \nu^{2}\left(2 \mu^{2}+\nu^{2}\right)}{(N+1)(M-U+2)\left(\mu^{2}+\nu^{2}\right)} .
$$

Proof: The proof is omitted due to space limitations.

The unified expressions in the Theorem hold for arbitrary linear precoder/decoder design, number of transmit/receive antennas, number of streams, and Rician channel statistics (and in turn arbitrary residual SI cancellation capability).

\section{Performance Analysis}

In this section, we conduct Monte-Carlo simulations of the entire RF transmit/receive processing chain in order to assess the validity of the theoretical findings. Without loss of generality, consider a $16 \times 8$ multi-user MIMO Rician fading channel with mean $\frac{1}{2}$ and variance 1 . The distributions of the residual SI power with linear ZF precoding and decoding are depicted with different number of transmit/receive streams in Figure 1 and Figure 2. For the single-user MIMO case in Figure 1, the proposed theorem provides a near exact fit with respect to the empirical data. The proposed Theorem also yields a tight approximation of the experimental values for the multi-user MIMO case in Figure 2. In general, it should be noted that the goodness of the fit of the theoretical distribution with respect to the empirical results increases for smaller mean $\mu$, variance $\nu$, and information streams $U$. On the other hand, increasing the number of transmit and receive antennas, for example in the context of massive MIMO, improves the tightness of the fit.

\section{SUMMARY}

A rigorous study of the residual SI in the context of FD multi-user MIMO was provided. We considered the case where a reference FD multi-antenna node utilized linear processing in order to simultaneously function as a transmitter and a receiver with respect to multiple FD radios. The bi-directional fading channels were drawn from a complex Gaussian distribution with arbitrary mean and variance. We provided exact closedform expressions for the first and second positive moments of the residual SI over Rician fading channels. The method of moments was then applied to derive a novel Gamma distribution for the residual SI. The main result from this paper can greatly assist with the performance analysis and optimization of FD multi-user MIMO wireless systems. 


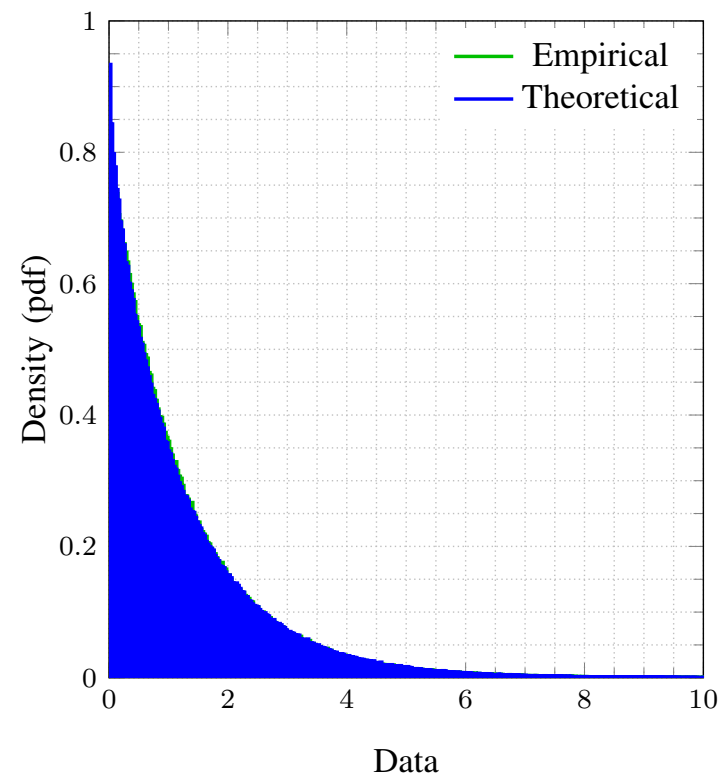

Figure 1. Simulation parameters are: $M=16, N=8, U=1$, $\mu=\frac{1}{2}, \nu=1$.

\section{REFERENCES}

[1] G. J. Foschini, "Layered space-time architecture for wireless communication in a fading environment when using multi-element antennas," Bell Labs Tech. J., vol. 1, no. 2, pp. 41-59, 1996.

[2] K.-K. Wong, R. D. Murch, and K. B. Letaief, "Performance enhancement of multiuser MIMO wireless communication systems," IEEE Trans. Commun., vol. 50, no. 12, pp. 1960-1970, Dec. 2002.

[3] F. Rusek, D. Persson, B. K. Lau, E. G. Larsson, T. L. Marzetta, O. Edfors, and F. Tufvesson, "Scaling up MIMO: Opportunities and challenges with very large arrays," IEEE Signal Process. Mag., vol. 30, no. 1, pp. 40-60, Jan. 2013.

[4] A. Shojaeifard, K.-K. Wong, M. Di Renzo, G. Zheng, K. A. Hamdi, and J. Tang, "Massive MIMO-enabled full-duplex cellular networks," arXiv preprint arXiv:1611.03854, 2016.

[5] C. X. Wang, F. Haider, X. Gao, X. H. You, Y. Yang, D. Yuan, H. M. Aggoune, H. Haas, S. Fletcher, and E. Hepsaydir, "Cellular architecture and key technologies for $5 \mathrm{G}$ wireless communication networks," IEEE Commun. Mag., vol. 52, no. 2, pp. 122-130, Feb. 2014.

[6] A. Sabharwal, P. Schniter, D. Guo, D. W. Bliss, S. Rangarajan, and R. Wichman, "In-band full-duplex wireless: Challenges and opportunities," IEEE J. Sel. Areas Commun., vol. 32, no. 9, pp. 1637-1652, Sept. 2014.

[7] J. G. Andrews, S. Buzzi, W. Choi, S. V. Hanly, A. Lozano, A. C. K. Soong, and J. C. Zhang, "What will 5G be?" IEEE J. Sel. Areas Commun., vol. 32, no. 6, pp. 1065-1082, June 2014.

[8] J. I. Choi, M. Jain, K. Srinivasan, P. Levis, and S. Katti, "Achieving single channel, full duplex wireless communication," in Proc. Annu. Int. Conf. on Mobile Computing and Networking (Mobicom), Sept. 2010.

[9] M. Jain, J. I. Choi, T. Kim, D. Bharadia, S. Seth, K. Srinivasan, P. Levis, S. Katti, and P. Sinha, "Practical, real-time, full duplex wireless," in Proc. Annu. Int. Conf. on Mobile Computing and Networking (Mobicom), Sept. 2011.

[10] M. Duarte, C. Dick, and A. Sabharwal, "Experiment-driven characterization of full-duplex wireless systems," IEEE Trans. Wireless Commun., vol. 11, no. 12, pp. 4296-4307, Dec. 2012.

[11] E. Everett, A. Sahai, and A. Sabharwal, "Passive self-interference suppression for full-duplex infrastructure nodes," IEEE Trans. Wireless Commun., vol. 13, no. 2, pp. 680-694, Feb. 2014.

[12] M. Chung, M. S. Sim, J. Kim, D. K. Kim, and C. b. Chae, "Prototyping real-time full duplex radios," IEEE Commun. Mag., vol. 53, no. 9, pp. 56-63, Sept. 2015.

[13] E. Everett, M. Duarte, C. Dick, and A. Sabharwal, "Empowering fullduplex wireless communication by exploiting directional diversity," in

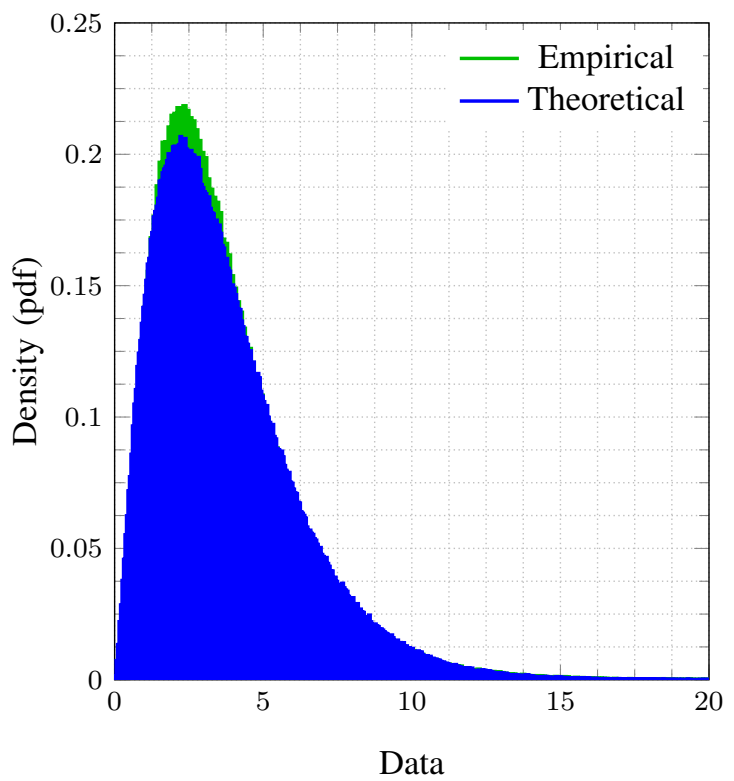

Figure 2. Simulation parameters are: $M=16, N=8, U=3$, $\mu=\frac{1}{2}, \nu=1$.

Proc. Asilomar Conf. Signals, Syst. and Comput. (ASILOMAR), Nov. 2011.

[14] B. Yin, M. Wu, C. Studer, J. R. Cavallaro, and J. Lilleberg, "Full-duplex in large-scale wireless systems," in Proc. Asilomar Conf. Signals, Syst. and Comput. (ASILOMAR), Nov. 2013.

[15] L. Anttila, D. Korpi, V. Syrjl, and M. Valkama, "Cancellation of power amplifier induced nonlinear self-interference in full-duplex transceivers," in Proc. Asilomar Conf. Signals, Syst. and Comput. (ASILOMAR), Nov. 2013.

[16] E. Ahmed, A. M. Eltawil, and A. Sabharwal, "Rate gain region and design tradeoffs for full-duplex wireless communications," IEEE Trans. Wireless Commun., vol. 12, no. 7, pp. 3556-3565, July 2013.

[17] Z. Zhang, X. Chai, K. Long, A. V. Vasilakos, and L. Hanzo, "Full duplex techniques for 5G networks: self-interference cancellation, protocol design, and relay selection," IEEE Commun. Mag., vol. 53, no. 5, pp. 128-137, May 2015.

[18] D. Bharadia and S. Katti, "Full duplex MIMO radios," in Proc. USENIX Symp. Networked Syst. Design and Implementation (NSDI), 2014.

[19] D. Kim, H. Lee, and D. Hong, "A survey of in-band full-duplex transmission: From the perspective of PHY/MAC layers," IEEE Commun. Surveys Tuts., vol. 17, no. 4, pp. 2017-2046, Fourth Quart. 2015.

[20] H. Q. Ngo, H. A. Suraweera, M. Matthaiou, and E. G. Larsson, "Multipair full-duplex relaying with massive arrays and linear processing," IEEE J. Sel. Areas Commun., vol. 32, no. 9, pp. 1721-1737, Sept. 2014.

[21] T. Riihonen, S. Werner, and R. Wichman, "Mitigation of loopback selfinterference in full-duplex MIMO relays," IEEE Trans. Signal Process., vol. 59, no. 12, pp. 5983-5993, Dec. 2011.

[22] Z. Zhang, Z. Chen, M. Shen, and B. Xia, "Spectral and energy efficiency of multipair two-way full-duplex relay systems with massive MIMO," IEEE J. Sel. Areas Commun., vol. 34, no. 4, pp. 848-863, Apr. 2016.

[23] I. Atzeni and M. Kountouris, "Full-duplex MIMO small-cell networks: Performance analysis," in Proc. IEEE Global Commun. Conf. (GLOBECOM), 2015.

[24] A. Shojaeifard, K. A. Hamdi, E. Alsusa, D. K. C. So, and J. Tang, "Exact SINR statistics in the presence of heterogeneous interferers," IEEE Trans. Inform. Theory, vol. 61, no. 12, pp. 6759-6773, Dec. 2015.

[25] A. Shojaeifard, K. A. Hamdi, E. Alsusa, D. K. C. So, J. Tang, and K. K. Wong, "Design, modeling, and performance analysis of multi-antenna heterogeneous cellular networks," IEEE Trans. Commun., vol. 64, no. 7, pp. 3104-3118, July 2016. 